\section{$\underset{\substack{\text { hommes } \\ \text { \& migrations }}}{ }$}

\section{Hommes \& migrations}

Revue française de référence sur les dynamiques

migratoires

1289 | 2011

Les frontières du sport

\title{
La conquête d'un espace de combat en milieu colonial
}

Le rugby sur les hautes terres de Madagascar (1896-1960)

\section{Évelyne Combeau-Mari}

\section{(2) OpenEdition}

Journals

Édition électronique

URL : https://journals.openedition.org/hommesmigrations/790

DOI : 10.4000/hommesmigrations.790

ISSN : 2262-3353

Éditeur

Musée national de l'histoire de l'immigration

Édition imprimée

Date de publication : 1 janvier 2011

Pagination : 20-27

ISSN : 1142-852X

Référence électronique

Évelyne Combeau-Mari, « La conquête d'un espace de combat en milieu colonial », Hommes \& migrations [En ligne], 1289 | 2011, mis en ligne le 31 décembre 2013, consulté le 21 septembre 2021.

URL : http://journals.openedition.org/hommesmigrations/790 ; DOI : https://doi.org/10.4000/

hommesmigrations.790 


\section{La conquête \\ d'un espace de combat en milieu colonial}

\section{Le rugby sur les hautes terres de Madagascar (1896-1960)}

Par Évelyne Combeau-Mari, professeur d'histoire contemporaine, université de La Réunion, CRESOI-EA12

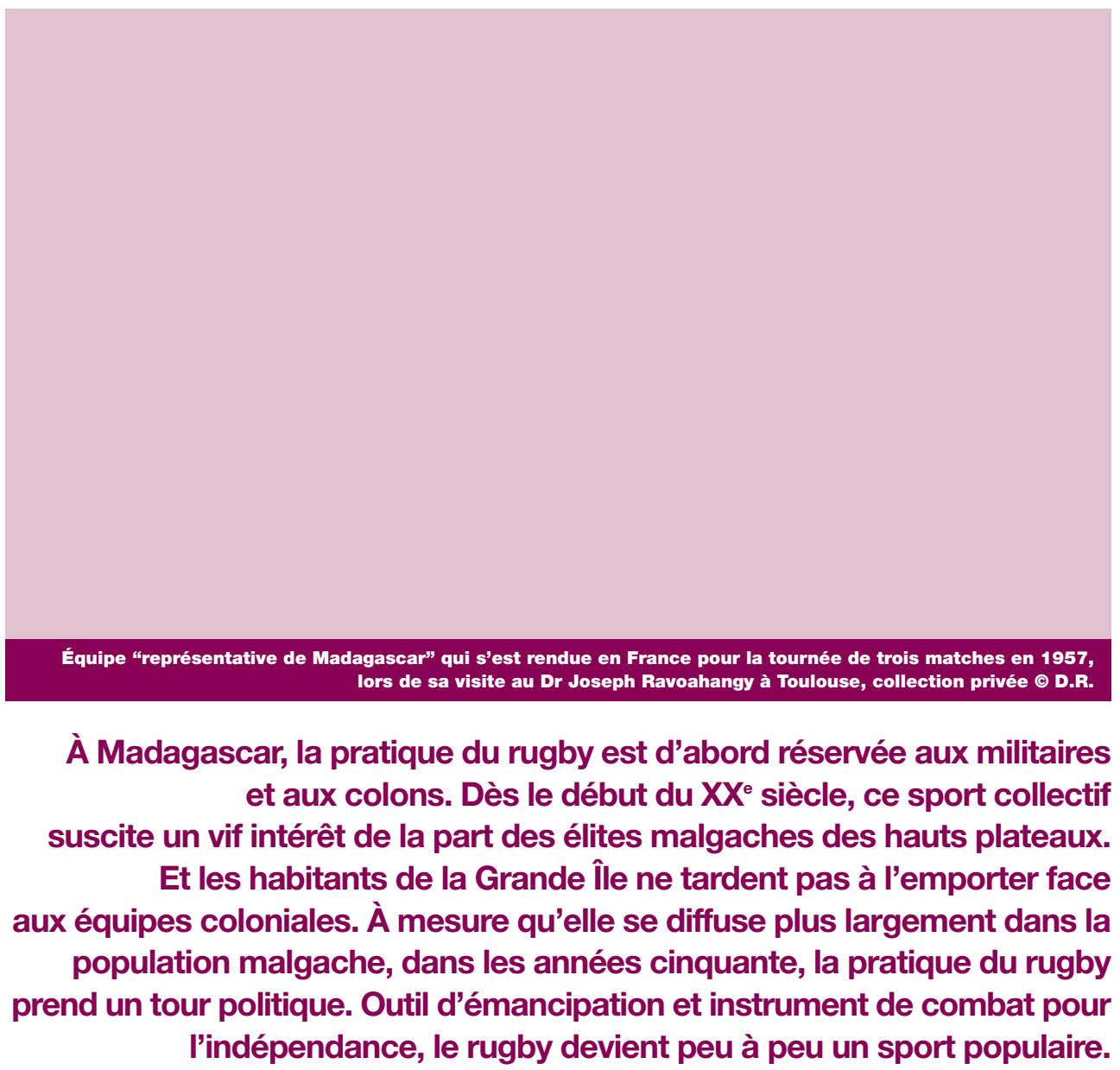


Inventé par les collégiens de la Rugby School au début du XIX siècle en Angleterre, le rugby incarne un nouveau modèle éducatif destiné aux futurs cadres pour forger des qualités d'endurance, de sang-froid, d'initiative, de solidarité et de sacrifice. Malgré les réticences des pédagogues français, il pénètre l'Hexagone grâce à la promotion des résidents anglais et à la réception enthousiaste des lycéens et des milieux anglophiles. Importé à Madagascar avec la colonisation française et les troupes du général Gallieni en 1896, le rugby, au même titre que les autres pratiques sportives, est réservé dans un premier temps exclusivement aux militaires et aux colons dans un but d'entraînement et de divertissement, alors que la gymnastique est censée préparer physiquement et moralement les jeunes Malgaches à la conscription. Or, dès le lendemain de la Première Guerre mondiale, les élites des hauts plateaux délaissent l'austère "méthode française" pour se focaliser sur la pratique du rugby et l'ériger en support d'émancipation nationale.

Il s'agit de revenir sur ce processus original de transfert culturel dans la période 18961960 qui couvre la colonisation française à Madagascar. Par "transfert culturel”, nous entendons observer les mécanismes de migration des pratiques sportives d'Europe vers cette île de l'océan Indien. Mais il s'agit également d'évaluer dans quelles conditions historiques la greffe du sport anglo-saxon se réalise et les motifs qui justifient l'appropriation spécifique par les populations des hautes terres, les Mérinas, d'un sport collectif comme le rugby dès la période de l'entre-deux-guerres. Transfert culturel et social car, au moment de l'indépendance, la démocratisation du jeu s'accompagne de la désertion de la frange éduquée de la population.

Cette démocratisation permet d'analyser les mécanismes de démarcation sociale dans l'accès au jeu. Au sein d'une société hiérarchisée en castes, elle souligne l'abandon par les élites mérinas de ce sport collectif de combat au profit des classes serviles.

\section{De l'importation du rugby à son appropriation (1896-1933)}

Dès le début du $\mathrm{XX}^{\mathrm{e}}$ siècle, dans le cadre de leurs activités d'endurcissement, les militaires français du bataillon d'occupation de l'Émyrne descendent tous les matins sur le champ d'entraînement pour se livrer entre autres sports au rugby. L'engagement et la violence du combat pour capturer le ballon fascinent les jeunes Malgaches qui, impatients de participer, deviennent très assidus sur le bord du terrain. À partir de 1905, des matches opposent une équipe formée de militaires, dénommée le Bataillon, à une équipe entièrement composée d'éléments locaux ${ }^{(1)}$ surnommés les Zanaky ni maraina, les "fils du matin", car ils s'entraînent au lever du jour sur l'esplanade de 
Mahamasina. Au regard du public de ces manifestations, les joueurs pressentent l'espace d'expression qui pourrait s'ouvrir à eux. Mais, dans l'organisation coloniale, les sports anglo-saxons ne sont accessibles qu'aux Français. En vertu du principe de préparation militaire des populations colonisées, les Malgaches doivent être formés à la défense de la patrie par l'apprentissage de la gymnastique. En phase avec le projet politique d'assimilation, l'administration initie la création de sociétés gymniques dans la capitale et en province.

La naissance de la société gymnique du Stade olympique de l'Émyrne le 16 décembre 1911 à Tananarive, présidée par l'administrateur Montagné, marque une étape importante dans l'affirmation du sport malgache. À la différence du Sportclub, réservé exclusivement aux Européens, le Stade ouvre largement ses portes aux Malgaches ${ }^{(2)}$. Encadrés par des personnalités d'exception telles que M. Montagné, ancien du Stade toulousain, et Georges Peyroutou, demi d'ouverture de l'équipe de France, les jeunes sont à bonne école et assimilent rapidement les fondamentaux du jeu. Peu motivés par la gymnastique militaire, ils l'abandonnent bientôt au profit du seul sport collectif de combat. Malgré cette exceptionnelle conjoncture, l'adhésion au rugby peut surprendre, d'autant qu'il s'agit d'un

Pratique distinctive, le rugby identifie les protestants, là où le football désigne les catholiques. cas unique en Afrique. Quelques éléments d'analyse autorisent une meilleure compréhension de la logique de diffusion.

Totalement étranger à la culture corporelle malgache, l'ovale présente néanmoins des caractéristiques qui rappellent les pratiques physiques des hauts plateaux. La violence de l'attaque pour la conquête du ballon répond aux exigences viriles des jeunes originaires de l'Imerina. De nombreuses activités ancestrales reposent sur le combat individuel, à l'image du diamanga, variété de boxe française interdite par l'administration, et du savika, combat contre les zébus. La dimension rugueuse de l'activité colle aux coutumes terriennes et rurales de ces régions fondées sur la culture du riz et l'élevage du zébu. Mais il faut également relever la conversion majoritaire des Mérinas au protestantisme à la fin du XIX', sous l'influence des missionnaires de la London Missionary Society, comme facteur de renouvellement des conceptions éducatives et culturelles. Très critiques à l'égard des jeux traditionnels considérés comme un reflet du paganisme, les pasteurs enseignent les valeurs occidentales au sein de l'Union de jeunesse. Pratique distinctive, le rugby identifie les protestants, là où le football désigne les catholiques. De plus, l'essence même du jeu a une connotation patriotique. La répartition des joueurs et l'organisation spatiale supposent de gagner du terrain sur l'adversaire tout en défendant son territoire contre l'envahisseur. 


\section{Quand les Malgaches l'emportent sur les colons}

Contre toute attente, en 1913 pour la première fois, l'équipe malgache du Stade olympique de l'Émyrne remporte une victoire sur l'équipe militaire ${ }^{(3)}$. Ce résultat fait l'effet d'une bombe dans les milieux coloniaux et retentit très fortement sur l'évolution du sport à Tananarive. Le sport malgache est lancé sur les hauts plateaux et se définit quasi exclusivement par la pratique du rugby. Les clubs quadrillent les différents quartiers de la ville. Répandue initialement par les étudiants de l'École de médecine et les jeunes scolarisés des secteurs privilégiés de la capitale, l'activité de combat collectif déplace progressivement ses frontières pour conquérir des zones plus populaires.

Au-delà des matches interclubs, le véritable enjeu de la compétition réside dans l'affrontement du Stade ou du Sport Hova contre l'équipe du Racing Club ${ }^{(4)}$. Relève du Bataillon, ce club fondé en 1920, dont l'appellation résume la vocation élitiste et européenne, regroupe d'anciens licenciés de clubs métropolitains, enseignants, fonctionnaires, commerçants et quelques élèves du lycée Gallieni. Mis en échec à plusieurs reprises devant le Stade, les dirigeants humiliés finissent, face à la violence des rencontres, par capituler et s'effacer du championnat en 1933. Ce recul du colonisateur sur son propre terrain est interprété comme un signe éminemment symbolique. Blessés dans leur amour-propre, les dirigeants sportifs français développent, à compter des années quarante, des stratégies de contrôle de l'activité rugbystique avec pour ambition sa reconquête définitive. Le fonctionnement administratif restrictif atteint son apogée entre 1941 et $1942^{(5)}$ avec l'arrivée sur la Grande Île du gouverneur Annet, ardent propagandiste de la "Révolution nationale". Il prend des formes plus détournées dans les années cinquante, bien que l'insurrection de 1947 et sa répression violente aient profondément bouleversé la nature des relations entre la France et la Grande Île.

\section{La place de l'ovale dans l'émancipation nationale (1947-1960)}

En dépit des réticences des cadres européens, la nouvelle législation engendre une adhésion sans précédent. Les années cinquante, période d'apogée du combat politique, correspondent à l'âge d'or du mouvement sportif. Dans la capitale et sa région, le rugby s'affirme comme le "sport-roi". L'ovale domine les autres activités par ses effectifs et par ses résultats exceptionnels qui en font le spectacle populaire par excellence.

Dans le cadre rénové de l'Union française, se dessinent de nouvelles orientations pour la jeunesse et les sports avec la création d'un Commissariat général aux sports, piloté par 
un inspecteur de la Jeunesse et des Sports chargé de développer des politiques sportives. De plus, l'extension de la loi de 1901 à Madagascar par le décret du 13 mars et l'arrêté du 12 avril 1946 accorde à tous les citoyens la liberté de se réunir. Mais ces nouvelles mesures sont profondément infléchies par les conséquences des événements de 1947. Considérés comme de puissants relais du mouvement nationaliste, les clubs de rugby sont désormais ciblés par l'administration. Plusieurs projets convergent pour contrôler leur activité et minimiser leur impact. Le premier repose sur les institutions avec la création d'une ligue. Il provoque l'émergence du jeu à treize et s'accompagne d'une campagne de presse sévère pour baisser la cote de popularité aux yeux de l'opinion publique. L'administration envisage également une décentralisation des pratiques sportives pour remettre en cause l'hégémonie de la capitale.

Malgré de multiples tentatives pour s'opposer à la vitalité du rugby malgache et en maîtriser l'expression, l'administration coloniale ne peut que mesurer son impuissance. La dynamique associative confirme la détermination nationaliste et l'avancée vers l'indépendance.

En 1951, à sa création, la ligue compte 21 clubs qui, comme leurs noms l'indiquent, sont représentatifs de tous les quartiers de la ville. 8 évoluent dans la division d'excellence, 13 en division d'honneur. Ainsi, au début de la saison 1957, la ligue affiche 3000 licenciés, dont plus de 500 juniors ${ }^{(6)}$. Mais la puissance du comité et des clubs de rugby provient également de sa trésorerie. Le stade d'Antanimena joue à guichets fermés tous les dimanches après-midi. La presse annonce régulièrement le chiffre de 10000 spectateurs autour du stade. À la tête d'une manne financière considérable, les grands clubs disposent d'une certaine indépendance et du pouvoir de décision.

\section{L'entrée dans le jeu des populations déshéritées}

La massification des effectifs au cours de la décennie cinquante, liée à l'instrumentalisation politique du jeu, se réalise par le recrutement progressif des joueurs dans les quartiers plus déshérités de Tananarive. La migration géographique de l'ovale du stade Mahamasina vers le stade Malacam d'Antanimena (stade des cheminots), au sein d'un quartier beaucoup plus pauvre, induit naturellement un glissement social du recrutement avec bientôt une majorité de mainty, descendants d'esclaves ${ }^{(\gamma)}$. Du fait de son écrasant poids démographique à Tananarive, cette part de la population est systématiquement sollicitée dans le débat politique. Dans une société fondée sur le système des castes, il reste cependant inconcevable pour un andriana, noble de 
descendance royale, ou bien un hova, roturier, de côtoyer sur un même terrain, a fortiori physiquement, un mainty. Ainsi, le processus de démocratisation du rugby, notable à compter des années cinquante, entraîne inexorablement la désertion des élites qui abandonnent non seulement l'activité mais aussi son encadrement. Cette évolution, compréhensible au regard des fondements culturels et sociaux des populations des hauts plateaux, est régulièrement stigmatisée par les journalistes français ${ }^{(8)}$. Les autorités redoutent la violence des débordements des milieux populaires, habituellement bridés par l'ascendant des descendants royaux. Prenant modèle sur le rugby français du SudOuest et sur l'image idéalisée de la "grande famille", ils dénoncent "la trahison des clercs". Pour autant, seul le rugby enregistre des résultats aussi exceptionnels en compétition. Grâce aux moyens financiers de la ligue, les dirigeants rehaussent le niveau technique des joueurs en multipliant les échanges. En 1952, l'équipe du Paris Université Club (PUC) est invitée pour une tournée tous frais payés. La prestation des joueurs malgaches répond aux espérances du public survolté de Mahamasina. Les bons résultats incitent le comité directeur de la ligue à solliciter en 1953 auprès de la Fédération française de rugby la venue d'une équipe de haut niveau et non des moindres, l'équipe de France. Les résultats étonnants obtenus par la sélection de Madagascar le 2 août 1953 dans un stade Mahamasina comble (plus de 20000 personnes) défrayent la chronique. L'Équipe du mercredi 5 août 1953 annonce même en première page : "pour l'équipe de France, la sélection de Madagascar manque d'un cheveu de créer une surprise sensationnelle."

Car les "Rouge et Jaune" font preuve lors de ce match d'une vivacité sur le ballon et d'une détermination à toute épreuve. Consciente de la différence flagrante de gabarit ${ }^{(9)}$, la sélection de Madagascar excelle dans la stratégie défensive. Même si ce score peut s'expliquer par la reprise récente de l'équipe de France après trois mois d'inaction, ou bien même dans son attitude condescendante à l'égard d'un adversaire dont elle méconnaît la valeur. Reste que les joueurs locaux réalisent une partie extraordinaire. Le niveau technique et tactique de leur prestation est confirmé lors du match revanche du 16 août 1953. Aguerrie, l'équipe de France révise son jeu, profite de son potentiel avant et ne concède que six points à ses adversaires qui exercent une résistance héroïque.

\section{Le rugby comme symbole du combat politique}

La réussite du rugby malgache incite le comité directeur de la ligue à préparer encore plus activement son équipe dans la perspective d'une tournée en France. De 1953 à 1957, l'entraîneur national, Clément Dupont, anime à raison de deux mois et demi 
par an un stage de formation intensif. En septembre 1957,l'équipe "représentative du rugby à Madagascar" est prête pour un déplacement de trois matches: Toulon, Toulouse, Paris.

Accueillie chaleureusement par les équipes provinciales, l'équipe malgache remporte brillamment ses trois premiers matches. Une tournée sportive marquée néanmoins par son engagement politique : les dirigeants et joueurs profitent de leur passage à Toulouse pour rendre visite au docteur Joseph Ravoahangy Andrianavalona encore assigné à résidence dans cette ville. La presse française salue unanimement l'originalité du jeu et l'efficacité de l'équipe.

Le point d'orgue de la tournée se déroule au stade Jean-Bouin. Les Malgaches affrontent le Racing Club de Paris devant un parterre de spectateurs réduit aux quelques étudiants compatriotes qui ont tenu à faire le déplacement. L'équipe visiteuse s'incline cette fois sur le score sévère de 3-33. Mais la défaite est rendue plus amère encore par le décès du joueur Randriambahiny (dit "Mbahiny") lors de la seconde mi-temps du match. Souhaitant stopper en pleine course le trois-quarts aile du Racing, situé à 10 mètres de l'en-but malgache, Mbahiny tente la "torpille", une technique particulière de plaquage usitée à Madagascar, très efficace si elle est réussie. Le joueur se projette en pleine vitesse, crâne en avant, contre la tempe, la mâchoire ou la région carotidienne, causant un vertige, une paralysie momentanée des membres inférieurs ou une fracture de la mâchoire de son adversaire. Tout aussi dangereuse pour celui qui l'exécute, cette technique peut provoquer une hémorragie interne. Elle entraîne ce jour-là la mort de Mbahiny. Le joueur du Racing porteur du ballon, stoppé brutalement, se reprend, finissant sa course par un essai dans l'en-but malgache.

Le drame survenu en "terrain ennemi" dans le cadre de l'activité sportive la plus porteuse d'espoir dans le camp nationaliste est perçu par l'opinion publique malgache comme une provocation inacceptable. La presse s'empare de l'événement tragique, érigeant le rugbyman en "vaillant kamikaze" de la cause patriotique ${ }^{(10)}$. La dernière tournée de l'équipe de rugby "représentative de Madagascar" en France révèle dans toute son intensité le sens politique du combat incarné par la pratique de l'ovale sur les hautes terres de Madagascar.

\section{Conclusion}

Par rapport aux autres colonies anglaises et françaises en Afrique, l'implantation du rugby à Madagascar offre une situation très originale en matière de diffusion ludique. En effet, la pratique des sports par les autochtones y est très précoce. Le choix privilégié de l'ovale est remarquable car tous les autres pays africains retiennent le football. 
Réservés initialement aux militaires et aux colons pour la pratique de divertissements dans des clubs fermés, les jeux sportifs collectifs ont été utilisés pour rehausser le contenu de la préparation militaire que la France destinait aux Malgaches. Aussitôt après la Première Guerre mondiale, les jeunes hommes des hauts plateaux issus des milieux éduqués et protestants prennent goût à la pratique du rugby. Ils s'emparent de l'activité au point d'en évincer les Européens. À la fin des années trente, le rugby, diffusé par les élites mérinas, représente le "sport malgache" et les clubs sportifs, des lieux de préparation physique et morale à l'émancipation nationale. Ce processus de "transfert culturel" du rugby à Madagascar montre l'appropriation d'une activité occidentale par les élites à des fins essentiellement politiques.

Grâce à l'assouplissement du régime associatif, la diffusion du sport collectif de combat s'accentue dans les années cinquante, mobilisant les couches plus défavorisées de la population. Ce mécanisme de démocratisation de l'ovale devient encore plus flagrant à compter de 1960. Instrument de combat pour l'indépendance, le rugby intéresse les élites mérinas qui s'en détournent définitivement une fois l'indépendance acquise en 1960. Si bien que le jeu est progressivement investi par les couches les plus pauvres de la population. Dans les représentations, le jeu appartient désormais aux descendants d'esclaves qui le pratiquent à la limite de la violence, expression de leur souffrance sociale.

\section{Notes}

1. Quelques noms et surnoms sont restés attachés à cette première équipe : Robinson Diable, Ratsimba Gôsy, Ravelonandro... Voir Razafindralambo Raymond, Le Rugby malagasy, Antananarivo, ministère de la Culture et de l'Art révolutionnaire, 1987. Bibliothèque nationale de Madagascar, 79633 (691)RAZ.

2. En 1925 , le club compte 180 "indigènes" et 2 "Européens". Courrier du président du Stade olympique de l'Émyrne à M . le président de la Fédération des sociétés de sports athlétiques de Madagascar (FSSAM) de décembre 1925. Archives nationales de Madagascar (ANM), D130.

3. Erhel Roger, "Les sports à Tananarive en 1952", in Tananarive, essai sur ses origines, son développement, son état actuel, Tananarive, Imprimerie officielle, 1952, pp. 245-248.

4. Fondé le 18 décembre 1920 et présidé par M. Lafosse, le club comporte des noms restés célèbres : Duriau surnommé Ingahibesola ("le vieux chauve"), Malhomme, un géant de 1,85 m, Xénophon, Phédon, Franceschi, Schneider, les frères Zelon, Allain, Danet. ANM, D130.

5. Voir la presse gouvernementale et les articles de Charles Petit "pro-européens". "Les lauriers sportifs", in La Tribune de Madagascar et dépendances, 17 janvier 1942.

6. Razafindralambo Raymond, op. cit.

7. Randriamaro Jean-Roland, "L'expression du politique par le populaire : l'exemple du rugby à Madagascar",

Revue historique de l'océan Indien, $\mathrm{n}^{\circ}{ }^{1}$, "Dynamiques dans et entre les îles du sud-ouest de l'océan Indien

(XVII'-XX' ${ }^{e}$ siècles)", 2005, p. 314.

8. Actualités Sports, nْ 18 (première année) du 29 octobre 1953.

9. Maty, Actualités Sports, $\mathrm{n}^{\circ} 10$ du 2 septembre 1953.

10. Voir Razafindralambo Raymond, op. cit. 